\title{
Coronary Artery Malformations Presenting as Acute Coronary Syndromes: A Case Series
}

\author{
Laura Jani, Roxana Hodas, Elena Beganu, Lehel Bordi \\ Clinic of Cardiology, County Clinical Emergency Hospital, Tîrgu Mureș, Romania
}

\section{ABSTRACT}

Coronary artery malformations are rare congenital abnormalities, which present non-specific symptoms such as atypical chest pain, malignant arrhythmia, or sudden cardiac death. The proper diagnosis of these abnormalities in emergency conditions can be very difficult, and noninvasive imaging techniques, such as computed tomography or magnetic resonance imaging, along with the gold standard represented by invasive coronary angiography, remain the most frequently used modalities for diagnosing these rare cases. We present four cases of coronary anomalies represented by an abnormal origin of the coronary arteries from the coronary ostium, presenting in emergency conditions with symptoms of acute myocardial infarction, which were diagnosed by urgent angiography.

Keywords: coronary artery malformation, coronary ostium, congenital heart diseases

\section{ARTICLE HISTORY}

Received: April 14, 2017

Accepted: May 25, 2017

\section{CORRESPONDENCE}

\section{Roxana Hodas}

Str. Gheorghe Marinescu nr. 50

540136 Tîrgu Mureș, Romania

Tel: +40 265212111

E-mail: roxana.hodas@yahoo.ro

\section{INTRODUCTION}

Coronary artery malformations are rare congenital abnormalities, most of them consisting in an abnormal origin of a coronary artery, with or without clinical impact. ${ }^{1}$ Due to their non-specific symptoms, such as atypical chest pain, malignant arrhythmia, or sudden cardiac death, caused by the reduction of blood flow in the coronary artery having an abnormal origin, the proper diagnosis of these abnormalities in emergency conditions can be very difficult. ${ }^{2,3}$ Noninvasive imaging techniques, such as computed tomography (CT) or magnetic resonance imaging (MRI), along with the gold standard represented by invasive coronary angiography, remain the most frequently used modalities for diagnosing these rare cases. ${ }^{4}$ The therapeutic algorithm is represented by conservative treatment (avoidance of heavy physical burden, use of beta-blockers), coronary angioplasty, or surgical intervention in the most severe cases. ${ }^{5}$

In this paper we present four cases of coronary anomalies represented by an abnormal origin of the coronary arteries from the coronary ostium, presenting in emergency conditions with symptoms of acute myocardial infarction, which were diagnosed by urgent angiography.

The patients agreed to the publication of their data and the institution where the patients had been admitted approved the publication of the cases.

\section{CASE 1}

A 66-year-old female patient with a history of hypertension and diabetes, with recurrent episodes of chest pain, dyspnea, and fatigue in the past, was admitted to 
our department accusing intense angina and diaphoresis. Physical examination was normal, and electrocardiography showed sinus rhythm with negative $\mathrm{T}$ waves in the inferior leads DII, DIII, aVF. The laboratory tests revealed a minimal elevation of creatine phosphokinase (CK 359 U/L). Echocardiography parameters were in normal ranges. However, the left ventricle was hypertrophic, with a left ventricular ejection fraction of $53 \%$. Due to the presence of recurrent angina symptoms, an invasive angiography was performed in order to assess possible coronary lesions and revealed the separate origin of the left descendent artery (LAD) and left circumflex artery (LCX) from two different coronary ostia located in the left side of the Valsalva sinus (Figure 1A). The LCX presented an ostial lesion and the LAD multiple, non-significant lesions, while the right coronary artery (RCA) was hypoplastic (Figure 1B). The patient received antiplatelets, statins, and diuretics, and was recommended to avoid intensive physical exertion. At the 6-month follow-up, the evolution of the patient was favorable, with significant regression of the symptoms.

\section{CASE 2}

A 63-year-old female patient, without any cardiovascular history, presented to our emergency room with intensive chest pain started 10 hours prior to presentation, asso-

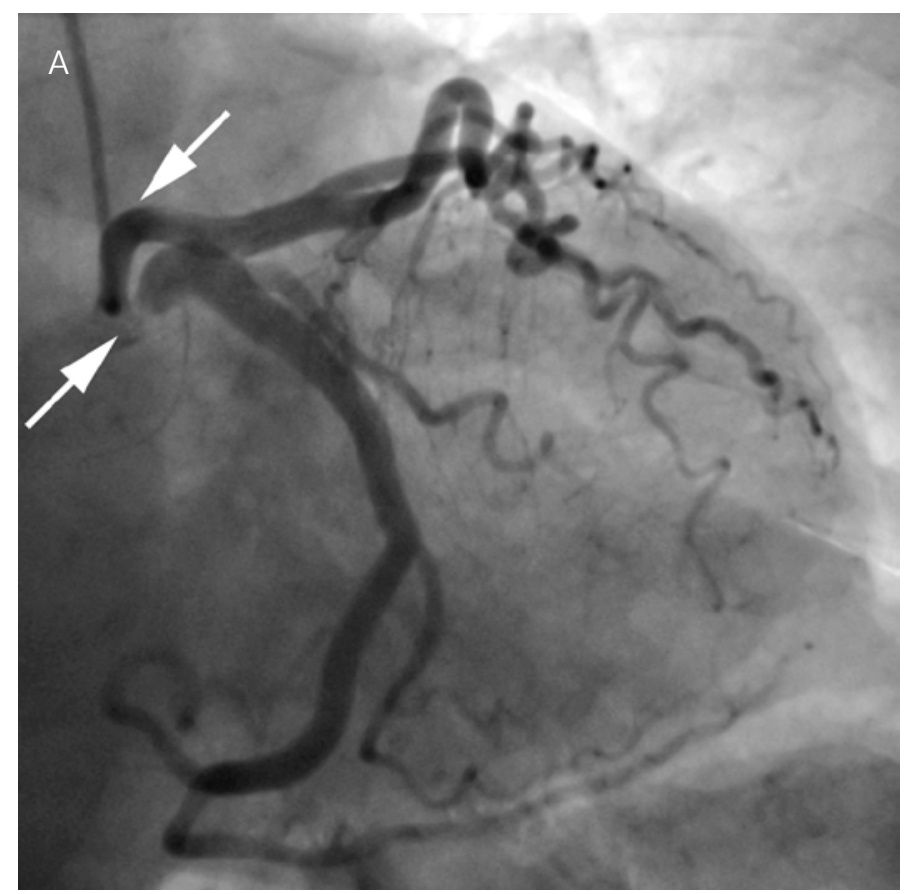

ciated with dyspnea and nausea. Electrocardiography revealed sinus rhythm and elevation of the ST segment with $2 \mathrm{~mm}$ in the right chest leads (V7, V8, V9). Coronary angiography performed in emergency revealed the common origin of the RCA and LCX from the right Valsalva sinus, with an acute thrombotic occlusion of the LCX (Figure 2A) and a severe lesion on the distal segment of the LAD. Two drug-eluting stents were implanted in the LCX and in the distal part of the LAD, with optimal TIMI III flow (Figure 2B). During the procedure the patient received $100 \mathrm{IU} / \mathrm{kg} /$ min heparin and the loading dose of eptifibatide, followed by continuous administration on the station. The next day after the intervention, she presented altered neurological status and obnubilation associated with diaphoresis. The emergency CT revealed a hemorrhagic stroke, therefore anticoagulation therapy was interrupted. During hospitalization, the cardiologic status remained stable. The patient was discharged with recommendations and a therapeutic plan (antiplatelet, statin, beta-blocker), and the neurological status of the patient presented a slow, but favorable evolution.

\section{CASE 3}

A 60-year-old male, smoker, with a history of diabetes and dyslipidemia, presented with unstable angina that was relieved by administration of nitroglycerin, associ-

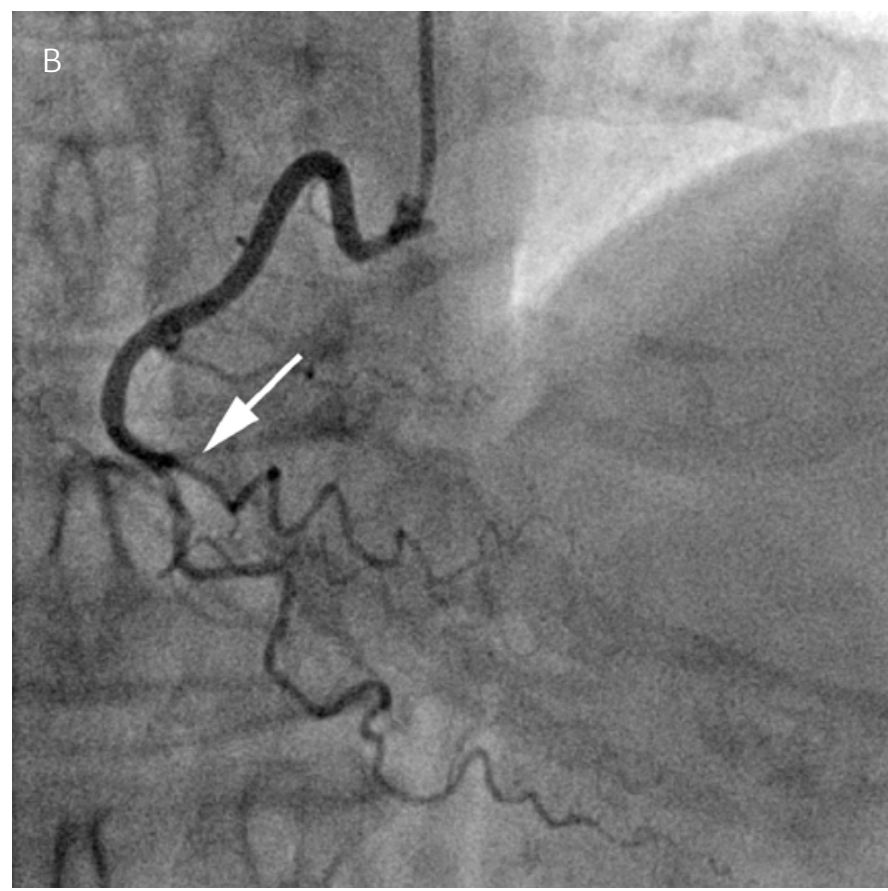

FIGURE 1. Case 1. A - Separate origin of the LAD and LCX from two different coronary ostia located in the left side of the Valsalva sinus (arrow); B - Hypoplastic RCA 

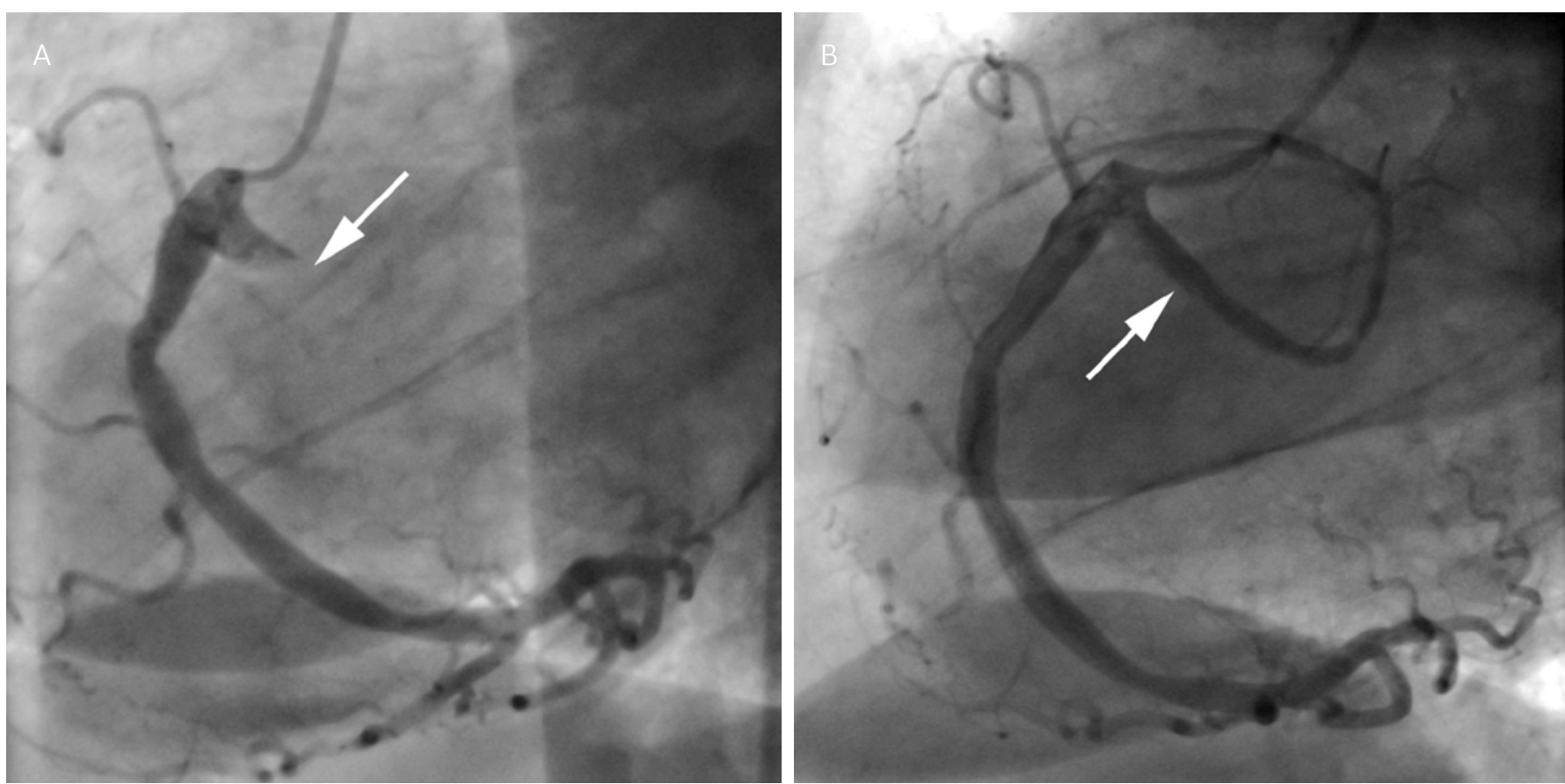

FIGURE 2. Case 2. A - Common origin of the RCA and LCX from the right Valsalva sinus with an acute thrombotic occlusion of the LCX (arrow); B - LCX recanalization after $\mathrm{PCl}$ and implantation of a drug eluting stent (arrow)

ated with vegetative symptoms. The patient reported that the chest pain started a week ago, during medium physical effort. The electrocardiogram showed negative $\mathrm{T}$ waves in the inferior leads, and echocardiography parameters were within normal ranges, with a good left ventricular ejection fraction. Coronary angiography showed the separate origin of the LAD and LCX, with lesions about $30-40 \%$ on the coronary arteries, without any hemodynamic significance
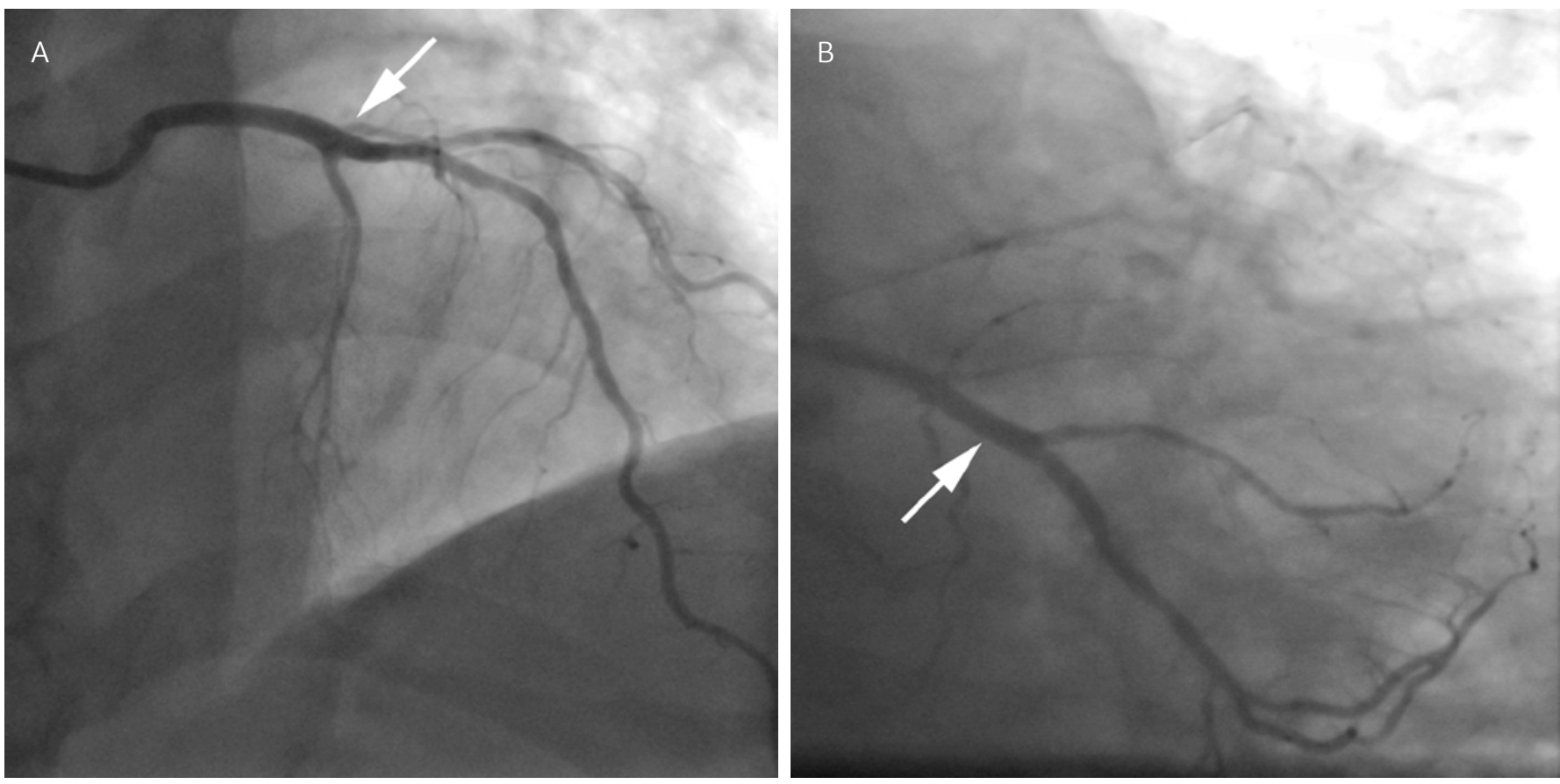

FIGURE 3. Case 3. Separate origin of the LAD and LCX from two different coronary ostia located in the left side of the Valsalva sinus. A - origin of LAD from a separate ostium (arrow); B - origin of the LCX from a separate ostium (arrow) 

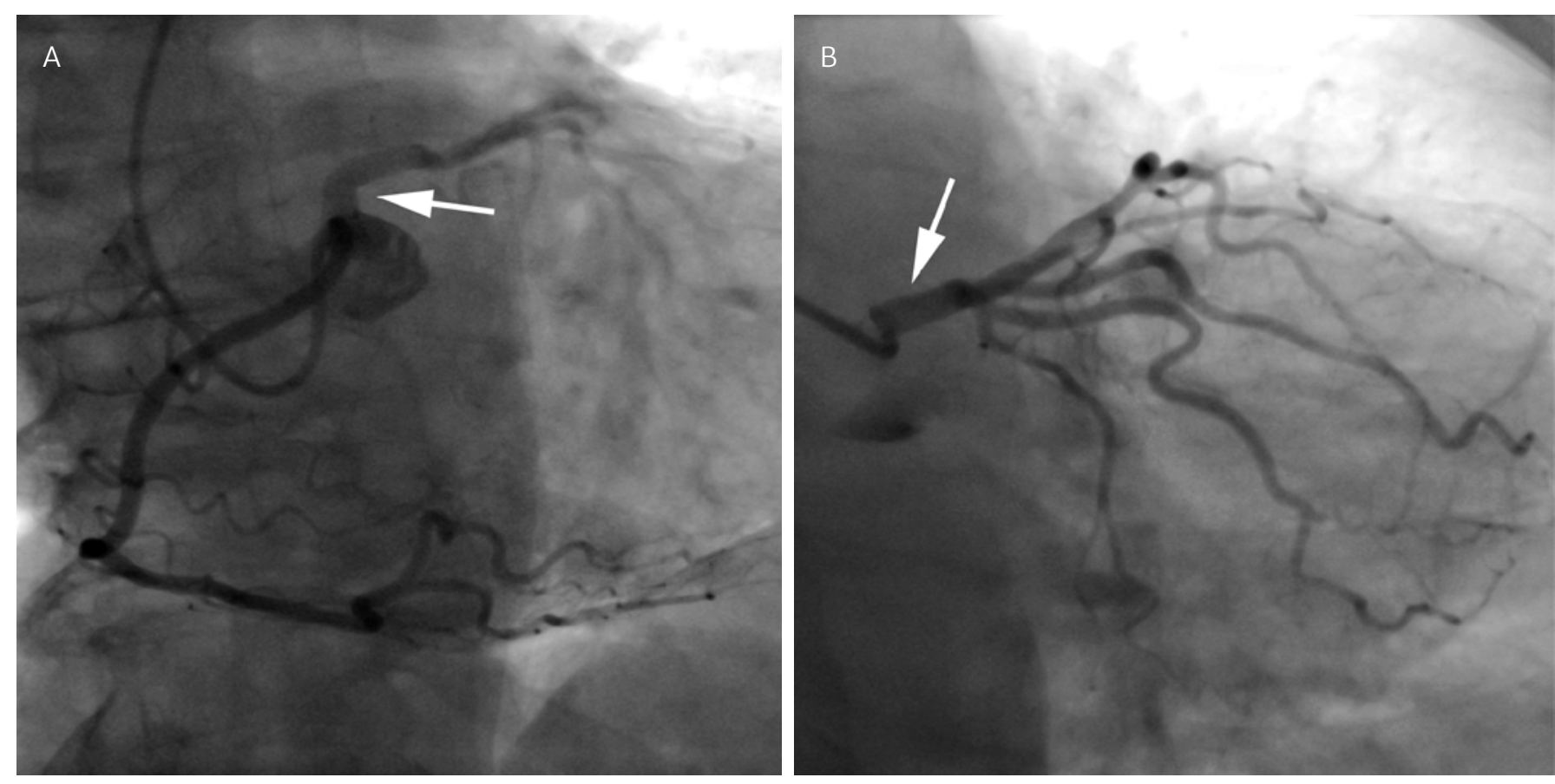

FIGURE 4. Case 4. A - Ectopic origin of the RCA from the left aortic sinus (arrow); B - No significant lesions on the LAD

(Figure 3A and 3B). Treatment consisted in beta-blockers and statins, and the patient presented a favorable evolution during regular medical follow-up.

\section{CASE 4}

The fourth case involves a 66-year-old, obese, smoker female patient, who presented at the emergency department with an intense retrosternal chest pain, started 7 hours prior to presentation. The electrocardiogram was not modified, and there were no elevations in serum levels of cardiac enzymes. Coronary angiography detected an ectopic origin of the RCA from the left aortic Valsalva sinus (Figure $4 \mathrm{~A}$ ) and no lesions on the LAD (Figure $4 \mathrm{~B}$ ). The patient received medical therapy and entered a rigorous follow-up program.

\section{DISCUSSIONS}

Coronary artery anomalies are rare forms of cardiac malformations that refer to the origin or the course of the artery. ${ }^{6}$ In most cases, they are underdiagnosed due to the lack of symptoms, and the detection occurs incidentally, in $0.3-0.6 \%$ of the cases during coronary angiography examination and about $1 \%$ on routine autopsy. ${ }^{2}$ The most frequent symptoms that can occur are thoracic chest pain due to myocardial hypoxia, myocardial ischemia, syncope, or malignant arrhythmias. This condition can be associ- ated with other cardiac diseases such as coronary artery lesions or heart valve disorders. ${ }^{3}$ Myocardial necrosis, congestive heart failure, or sudden cardiac death represent the most severe complications that can occur in this type of diseases. ${ }^{7}$

In certain cases, the diagnosis of this particular form can represent a challenge due to the fact that most patients are asymptomatic. This type of abnormality is usual associated with a poor tissular perfusion that can be responsible for hypoxia and myocardial ischemia. ${ }^{3}$ In these cases, hypoxia is related to intramural hypoplasia or to the external compression of the vessel. ${ }^{8}$ Whenever a coronary abnormality is diagnosed, it is extremely important to identify the exact origin of the abnormality and its relationships with other cardiac structures. The gold standard method for the diagnosis of these particular forms remains coronary angiography. Coronary CT offers the finest spatial resolution for the assessment of these lesions, being able to reveal the localization of the malformations and to assess their complexity. ${ }^{2,9}$ Magnetic resonance imaging also provides safe information; however, the small size of the vessels, the impact of breathing, or the rapid motion of the coronaries can represent limiting factors for this examination. ${ }^{10}$

In some cases of coronary anomalies that involve the right side, percutaneous interventions and stenting can be recommended in the presence of the following criteria: high risk for sudden death, significant intramural 
hypoplasia, documented reversible ischemia. The use of intravascular ultrasound before and during angioplasty is highly recommended in this type of cases. ${ }^{11}$

According to the literature, severe forms of coronary anomalies located in the left side of the heart might require surgical correction, consisting in re-implantation of the ectopic coronary in the aortic sinus, or osteoplasty, which creates a new ostium at the end of the intramural segment. ${ }^{12}$

All four patients presented in this case series were asymptomatic in their history and accused intense chest pain at presentation in the emergency room. Coronary angiography, indicated by the symptoms suggestive for acute myocardial infarction, identified the etiology of the chest pain and led to appropriate therapy in each case.

\section{CONCLUSIONS}

Coronary artery malformations are extremely rare abnormalities that are frequently underdiagnosed. Depending on the type of malformation and the associated lesions, the clinical presentation may vary from asymptomatic conditions to sudden cardiac death. As a consequence of the non-specific symptoms, the diagnosis can be challenging in many cases. Coronary angiography, as the gold standard imaging diagnostic technique, is able to reveal the type of malformation and its complexity, and can guide therapy in these rare diseases.

\section{CONFLICT OF INTEREST}

Nothing to declare.

\section{ABBREVIATIONS}

LAD left anterior descending artery

LCX left circumflex artery

RCA right coronary artery

PCI percutaneous coronary intervention

\section{REFERENCES}

1. Angellini P. Coronary Artery Anomalies An Entity in Search of an Identity. Circulation. 2007;115:1296-1305. doi: 10.1161/ CIRCULATIONAHA.106.618082.

2. Villa AD, Sammut E, Nair A, Rajani R, Bonamini R, Chiribiri A. Coronary artery anomalies overview: The normal and the abnormal. World J Radiol. 2016;8:537-555. doi:10.4329/wjr. v8.i6.537.

3. Yuan SM. Anomalous origin of coronary artery: taxonomy and clinical implication. Rev Bras Cir Cardiovasc. 2014;29:622629. doi:10.5935/1678-9741.20140109.

4. Marler AT, Malik JA, Slim AM. Anomalous Left Main Coronary Artery: Case Series of Different Courses and Literature Review. Case Rep Vasc Med. 2013;2013:380952. doi:10.1155/2013/380952.

5. Maron BJ, Zipes DP. Introduction: eligibility recommendations for competitive athletes with cardiovascular abnormalities general considerations. J Am Coll Cardiol. 2005;45:1318-1321. doi: 10.1016/j.jacc.2005.02.006.

6. Angelini P. Normal and anomalous coronary arteries: definitions and classification. Am Heart J. 1989;117:418-434.

7. Basso C, Maron BJ. Clinical profile of congenital coronary artery anomalies with origin from the wrong aortic sinus leading to sudden death in young competitive athletes. J Am Coll Cardiol. 2000;35:1493-1501.

8. Peñalver JM, Mosca RS, Weitz D, Phoon CK. Anomalous aortic origin of coronary arteries from the opposite sinus: a critical appraisal of risk. BMC Cardiovasc Disord. 20121;12:83. doi: 10.1186/1471-2261-12-83.

9. Montaudon M, Latrabe V, Iriart X, Caix P, Laurent F. Congenital coronary arteries anomalies: review of the literature and multidetector computed tomography (MDCT)-appearance. Surg Radiol Anat. 2007;29:343-355. doi: 10.1007/s00276007-0217-1.

10. Bunce $\mathrm{NH}$, Lorenz $\mathrm{CH}$, Keegan J. Coronary artery anomalies: assessment with free-breathing three-dimensional coronary MR angiography. Radiology. 2003;227:201-208. doi: 10.1148/ radiol.2271020316.

11. Angelini P, Velasco JA, Ott D, Khoshnevis GR. Anomalous coronary artery arising from the opposite sinus: descriptive features and pathophysiologic mechanisms, as documented by intravascular ultrasonography. J Invasive Cardiol. 2003;15:507-514.

12. Mustafa I, Gula G, Radley-Smith R, Durrer S, Yacoub M. Anomalous origin of the left coronary artery from the anterior aortic sinus: a potential cause of sudden death: anatomic characterization and surgical treatment. J Thorac Cardiovasc Surg. 1981;82:297-300. 